\title{
A COMPUTER SIMULATION OF THE MIDLATITUDE PLASMASPHERE AND IONOSPHERE
}

\author{
E. R. YOUNG, ${ }^{*}$ D. G. TORR, $\dagger$ P. RICHARDS $\dagger$ and A. F. NAGY \\ Space Physics Research Laboratory, The University of Michigan, Ann Arbor, MI 48109, U.S.A.
}

(Received in final form 22 April 1980)

\begin{abstract}
The main objective of this paper is to report the development of a new computer model to simulate species density, temperature and plasma flow in the ionosphere and plasmasphere. The paper provides a general description of the code for potential users. The model incorporates the attractive features of previous models reported in the literature. In addition significant improvements have been made, which render the code a comprehensive tool for studying the ionosphere-plasmasphere environment. For example, the calculations are carried out across an entire field tube extending from the $E$ region in one hemisphere to the $E$ region in the conjugate ionosphere. The coupled continuity, momentum, energy, heat flow and photoelectron equations are solved in a quasi-simultaneous manner. The efficiency of the numerical solution was much improved by using an integral form of the continuity and momentum equations which resembles the form used in the shooting method at high altitudes, and the conventional parabolic solution in the $F$ region and topside ionosphere below $2000 \mathrm{~km}$. The equations are formulated to facilitate linking the plasmasphere and ionosphere. The equations have been expressed in a new way which we have called "flux preserving," and solved using a Newton iteration. The speed of convergence of the solution procedure is further improved by the use of predictive techniques.

The results reported in the paper are the product of numerical tests that were carried out to assess the performance of the code. The choice of material for analysis therefore was not determined by geophysical criteria. However, several interesting results were obtained which warrant reporting. These include (1) the discovery that counterstreaming of $\mathrm{H}^{+}$and $\mathrm{O}^{+}$ions occurs under steady state conditions; (2) that our theoretical results agree with recent measurements of electron temperature in the plasmasphere, which are significantly lower than previous measurements. However, there is an indication that a magnetospheric source of heat may nevertheless be required for precise agreement.
\end{abstract}

\section{INTRODUCTION}

Study of the thermal plasma population in the closed field line region of the magnetosphere has grown in importance in recent years (see review by Chappell, 1975). Not only is there a significant interchange of plasma between the ionosphere and plasmasphere, but the thermal plasma has also been found to affect the energetic particle population of the magnetosphere. This tight coupling between the magnetosphere-ionosphere system via hot and cold plasma has an impact on energetic particles of the radiation belts, ring current particles and the plasma sheet. Although the broad features have been established (Carpenter and Smith, 1964) a realistic comprehensive model of the plasmasphere has not yet been developed.

* Currently at Research Center, Regis College, Weston, Massachusetts.

† Also affilitated with the National Institute for Telecommunications, Research of the C.S.I.R., Johannesburg, South Africa. Present Address: Center for Atmospheric and Space Sciences, Utah State University, UMC 41, Logan, UT 84322, U.S.A.
It seems clear that the interchange flow processes arc related to the shaping of the plasmapause and light ion trough features (Chappell, 1975). The global morphology of the plasmasphere varies strongly with magnetic activity and associated electric fields. When the convection electric field increases during magnetic storms the plasmasphere decreases in size owing to compression on the night side and a peeling off and convective loss of plasma in the afternoon dusk sector. During magnetically quiet periods, the plasmasphere increases in size, refilling as a result of upward flow of cold plasma from the ionosphere. One of the primary objectives of studies of ionospheric-magnetosphere coupling is to relate global storm and quiet time patterns of the behavior of the plasmasphere to those of the ionosphere.

A fundamental requirement for understanding the processes governing the ionosphereplasmasphere system is a knowledge of the concentration, temperature and flow velocities of charged species populating the field tubes. Since no experiment has yet been devised which can supply these parameters along the full extent of the field 
tube, we must, of necessity, resort to models to unify and interpret available and forthcoming fragments of data. Studies of field tube refilling constitute an important approach to the overall problem. Although many studies of this type have been carried out in recent years, the details of the filling process still remain unknown.

The problem of protonosphere-ionosphere coupling has been studied mainly from two complementary viewpoints, namely

(1) as a source of ionization in the nocturnal $F$ region.

(2) as a source and sink of plasma for the protonosphere.

From the protonospheric point of view proton escape serves to replenish field tubes that have lost their ionization, while from the ionospheric viewpoint, the protonosphere is a reservoir of ionization which is filled during the day, and which acts as a source of ionization and energy for the $F$ region at night. Several obvious discrepancies in the expected signature of the ionosphere on the plasmasphere lead to studies of proton filling rates in the plasmasphere due to outflow from the ionosphere (Banks et al., 1971). For example, the plasma density increases on the equatorward side of the light ion trough at $L$ values of 2 , whereas the plasmapause lies beteen $L=4-6$.

Early theoretical studies predicted significant fluxes of $\mathrm{O}^{+}$ions into the $F$ region via the charge exchange process of $\mathrm{H}^{+}$with $\mathrm{O}$ (Hanson and Patterson, 1964; Geisler and Bowhill, 1965; Geisler, 1967). These predictions were soon confirmed by experimental results (Vasseur and Waldteufel, 1968; Evans, 1969, 1971 a, b; Evans et al., 1970; Behnke, 1970; Hagen, 1972). Subsequent theoretical work prior to 1978 confirmed the importance of the protonosphere as a source of ionospheric plasma (cf. for example, Nagy et al., 1968; Banks et al., 1971; Schunk and Walker, 1972; Nagy and Banks, 1972; Schunk and Walker, 1973; Moffett and Murphy, 1973; Massa et al., 1974; Murphy et al., 1976). The studies have shed considerable light on filling processes. However, with the exception of Nagy et al. (1968) and Mayr et al. (1972) the results are all based on single hemisphere models.

The work of Park and Banks (1974) and Murphy et al. (1976) produced several major new results. The former studied the effects of plasma flow into the ionosphere under the influence of neutral winds and an east-west electric field. The latter studied field tube filling after substorm depletion and the subsequent effects on the plasmasphere and ionos- phere. Bailey et al. (1978) repeated the calculations of Murphy et al. (1976) for sunspot maximum conditions.

A special study of interest was reported by Bailey et al. (1977). They used the one hemisphere model to theoretically confirm the counterstreaming of $\mathrm{O}^{+}$and $\mathrm{H}^{+}$ions observed by Vickrey et al. (1976) at twilight from Arecibo. This topic is discussed further for a more general case in the section reporting results obtained with the current model.

Relatively few theoretical studies have treated the interhemispheric flow of plasma, despite the fact that the phenomenon may be important. Rothwell (1963), Hanson (1964) and Kohl (1966) discussed interhemispheric flow in connection with the $F_{2}$ layer winter anomaly. Nagy et al. (1968) studied interhemispheric flow as a source of heat for the local topside ionosphere. Recent models which included interhemispheric flow were developed by Mayr et al. (1972), Kutimskaya et al. (1973), Bailey et al. (1978). Murphy and Moffett (1978) used a single hemisphere model to assess the effects of interhemispheric flow. Bailey et al. (1978) extended their earlier model to two hemispheres, and Murphy and Moffett (1978) recently included the energy equations, and nonlinear acceleration terms in the momentum equations.

\section{FORMULATIONS AND SOLUTIONS OF THE TRANSPORT EQUATIONS}

Despite significant advances over the last decade, which were mentioned above as well as in a number of reviews (Carpenter and Park, 1973; Chappell, 1972; Banks, 1972; Banks et al., 1976), the plasma coupling mechanisms between the ionosphere and magnetosphere have not been studied using a formulation which simultaneously accounts for variations in all the major controlling variables. Schunk $(1975,1977)$ points out that the basic equations used in modelling the ionosphere and protonosphere have not, in general, been applied with a clear recognition of their intrinsic limitations.

We have adopted the modified momentum equations of St. Maurice and Schunk (1977) and energy equations of Schunk and Nagy (1978) as appropriate for our current concepts of the quiet plasmasphere (Schunk and Watkins, 1979). Application of this system to the midlatitude ionosphere and plasmasphere permits several simplifiying assumptions to be made which significantly reduce the complexity of the equations.

We consider a plasma comprised of only two 
major ions, $\mathrm{O}^{+}$and $\mathrm{H}^{+}$and electrons and an arbitrary number of minor ions in a neutral atmosphere of $\mathrm{O}, \mathrm{O}_{2}, \mathrm{~N}_{2}$ and $\mathrm{H}$. We assume that species temperature and flow velocity differences are small, a midlatitude condition. Thus we can neglect stress and nonlinear acceleration terms, and use Burgers' (1969) linear collision terms ( $c f$. St. Maurice and Schunk, 1977). In addition, density and temperature gradients perpendicular to the geomagnetic field lines are neglected, and we have assumed that ion and electron temperature distributions are isotropic so that we can ignore the stress tensor equation.

Even though many of the individual terms in our equations arise directly from Schunk's 13 -moment system of equations, we have arrived finally at a basic formulation involving only six equations. These are: the photoelectron two stream equation, the electron energy equation, the ion energy equation, St. Maurice and Schunk's (1977) velocity solution of the ion and electron momentum equations, and finally the continuity equations. We have included ion neutral collisions in the equations of St. Maurice and Schunk in order to solve for the individual ion velocities separately. These basic equations of our plasma formulation are given collectively in (1-6) in the section on Basic Equations.

The primary photoelectrons are treated via a two stream Liouville equation (Banks and Nagy, 1970) which essentially follows their distribution through phase space until they are thermalized. One hundred energy steps are used at each spatial grid point separately for the upstreaming and downstreaming electron populations. Such a detailed phase space treatment is necessary only for the highly superthermal photoelectrons.

Currently neutral winds can be input numerically, or the code could be modified to include an analytical fit to experimental or model wind values. Provision has not yet been made for electric field convection of tubes of flux.

A major shortcoming in previous studies has been the use of ad hoc boundary conditions, which generally arises when only part of a field line is modelled. Roble (1975) and Stubbe (1970) formulated their coupled parabolic equations for $\mathrm{O}_{2}{ }^{+}$, $\mathrm{NO}^{+}, \mathrm{O}^{+}$and $\mathrm{H}^{+}$in the $F$ region and topside ionosphere, but $\mathrm{H}^{+}$was treated as a minor ion in each of these cases, or the $\mathrm{H}^{+}$density is calculated by assuming chemical equilibrium with $\mathrm{O}^{+}$. Such formulations all apply to altitudes below about $2000 \mathrm{~km}$, and require artificially designated or measured upper boundary conditions.

Bauer (1968) and Massa (1974) attempted to extend this treatment for $\mathrm{O}^{+}$and $\mathrm{H}^{+}$along the entire flux tube but this leads to serious numerical problems, which are discussed in the next section. The so called shooting method has also been used in attempts to treat the entire flux tube by a single method. In the shooting method (Moffett and Murphy, 1973; Mayr et al., 1967; Murphy and Moffett, 1978; Bailey et al., 1977; Richards, 1978) the flux or velocity from the continuity equation is substituted into the collision terms of the momentum equation. The resulting intergro-differential equation is then integrated numerically down from the equator where flux and density boundary conditions are imposed. The equatorial boundary conditions are readjusted and the integration performed repeatedly until low altitude chemical boundary conditions are satisfied. The main disadvantages of this approach stem from the fact that flux determinations from the continuity equation are inherently inaccurate and unstable at low altitudes, and the method does not produce simultaneous solutions in both hemispheres. We have compared our method with the shooting method approach of Richards (1978). The computer time required to achieve convergence is about an order of magnitude smaller using our method.

The perturbation solution of Mayr et al. (1972) also treats the entire field line as a single region. Mayr et al. integrate numerically but with only a gradually increasing fraction of the collisional terms in the momentum equations, along the entire field line. The drag terms are always evaluated in the $n$th approximation to obtain the $n+1$ st approximation. This method also suffers from low altitude problems for the same reasons mentioned in connection with shooting methods. It is apparent that both of these methods are basically high altitude formulations and that neither should be used unless an accurate lower boundary at, say, $1000 \mathrm{~km}$ can be supplied by some other type of calculation or by measurement.

Multiple region formulations somewhat similar to our own have been used in simpler form by Park and Banks (1974) and Marubashi and Grebowsky (1976), who treated the topside region above $3000 \mathrm{~km}$ as a reservoir.

We surmount the limitations discussed above in our treatment by simulating the flow and density in an entire flux tube spanning the midlatitude plasmasphere between magnetically conjugate points in the $F$ regions of the northern and southern ionospheres, as shown in Fig. 1. The solution emcompasses several regions of differing dominant physical processes. 


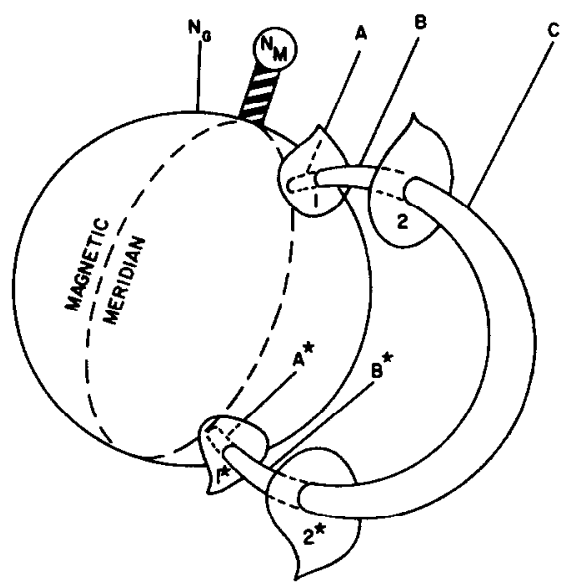

Fig. 1. Regions and Boundaries.
A, $A^{*}$
Regions of local chemistry.
$\mathrm{B}, \mathbf{B}^{*}$
Ion diffusion regions where parabolic density equations can be formulated.
C Diffusive equilibrium region.
$1,1^{*}, 2,2^{*}$ Boundaries between adjacent reg- ions: at 1 and $1^{*}$ densities are con- tinuous, while at 2 and $2^{*}$ both den- sity and flux are continuous.

In order to provide a geophysically meaningful and consistent simulation, a number of parameters must be computed in the proper sequence as shown in Fig. 2 which is an overall schematic of our simulation code. Neutral atmospheric parameters are calculated locally from the MSIS model atmosphere (Hedin et al., 1977a, b) because it reproduces the seasonal solar cyclic and diurnal variations of the atmosphere as well as its response to geomagnetic disturbances. Solar fluxes of $\mathrm{M}$. Torr et al. (1979), Hinteregger (1978) and photoionization cross sections listed by Kirby-Docken et al. (1979) are used. Most recent rate coefficients given by St. Maurice and Torr (1978) and D. Torr and Torr (1979) are used.

\section{BASIC EQUATIONS}

Photoelectron Liouville equation

$$
\begin{aligned}
& B \frac{\mathrm{d}}{\mathrm{d} s} \frac{\Phi^{+}}{B}=-T_{2} \phi^{+}+T_{1} \phi^{-}+\frac{q}{2\langle\cos \theta\rangle}+\frac{q^{+}}{\langle\cos \theta\rangle}, \\
& -B \frac{\mathrm{d}}{\mathrm{d} s} \frac{\Phi^{-}}{B}=-T_{2} \phi^{-}+T_{1} \phi^{+}+\frac{q}{2(\cos \theta\rangle}+\frac{q^{-}}{\langle\cos \theta\rangle},
\end{aligned}
$$

where

$\phi^{+}(E, s)=$ photoelectron flux outward along $s$
$\phi^{-}(E, s)=$ photoelectron flux inward along $s$

$q(E, s)=$ photoelectron production rate in the range $E$ to $E+\mathrm{d} E$ due to direct ionization processes.

$q^{ \pm}=$photoelectron production in the range $E$ to $E+\mathrm{d} E$ due to cascading from higher energy photoelectrons undergoing inelastic collisions

$\langle\cos \phi\rangle=$ average cosine of pitch angle

$B=$ magnetic field strength

$T_{1}=\sum_{k} n_{k} p_{e}^{k} \sigma_{e}^{k}$

$$
T_{2}=\sum_{k} n_{k}\left[{\sigma_{a}}^{k}+p_{e}{ }^{k} \sigma_{e}{ }^{k}\right]
$$

and

$$
\begin{aligned}
n_{k}= & k \text { th species number density } \\
p_{e}{ }^{k}= & \text { photoelectron backscatter probability for } \\
& \text { elastic conditions with the } k \text { th species. } \\
\sigma_{e}{ }^{k}= & \text { photoelectron total scattering cross- } \\
& \text { section for elastic conditions with the } \\
& k \text { th species. } \\
\sigma_{\mathrm{a}}{ }^{k}= & \text { inelastic cross-section for excitation of } \\
& \text { the } k \text { th particle species. }
\end{aligned}
$$

Thermal electron energy

$$
\begin{aligned}
{ }_{\frac{3}{2}}^{\frac{3}{2}} N_{e} k \frac{\partial T_{\mathrm{e}}}{\partial t}= & -N_{e} k T_{e} \nabla \cdot U_{e} \\
& -\frac{3}{2} N_{\mathrm{e}} k U_{\mathrm{e}} \cdot \nabla T_{\mathrm{e}}-\nabla \cdot q_{\mathrm{e}}+\Sigma Q_{\mathrm{e}}-\Sigma L_{\mathrm{e}} .
\end{aligned}
$$

Ion energy

${ }_{2}^{3} N_{1} \mathrm{k} \frac{\partial T_{\mathrm{i}}}{\partial t}=-N_{\mathrm{i}} k T_{\mathrm{i}} \nabla \cdot U_{\mathrm{i}}$

$$
-\frac{3}{2} N_{\mathrm{i}} k U_{\mathrm{i}} \cdot \nabla T_{\mathrm{i}}-\nabla \cdot q_{\mathrm{i}}+\Sigma Q_{\mathrm{i}}-\Sigma L_{\mathrm{i}} .
$$

Ion heat flow

$$
q_{\mathrm{i}}=\frac{1}{1-\xi}\left(-\lambda_{\mathrm{i}} \nabla T_{\mathrm{i}}-\frac{N_{\mathrm{i}} m_{\mathrm{i}}}{N_{\mathrm{j}} m_{\mathrm{j}}} \frac{\nu_{\mathrm{ij}}^{\prime}}{\nu_{\mathrm{j}}^{\prime}} \lambda_{\mathrm{i}} \nabla T_{\mathrm{i}}\right) .
$$

Electron heat flow

$$
q_{\mathrm{e}}=-\lambda_{\mathrm{e}} \nabla T_{\mathrm{e}} .
$$

Ion electron momentum

$$
\begin{aligned}
& {\left[\begin{array}{l}
\phi_{1} \\
\phi_{2}
\end{array}\right]=-\left[\begin{array}{cc}
N_{1} & 0 \\
0 & N_{2}
\end{array}\right]\left[\begin{array}{cc}
\left(1+\frac{\nu_{2 x}}{\nu_{21}}\right) & 1 \\
1 & \left(1+\frac{\nu_{1 x}}{\nu_{12}}\right)
\end{array}\right]} \\
& \times\left[\begin{array}{ll}
D_{1} & Q_{1} \\
D_{2} & Q_{2}
\end{array}\right]\left[\frac{\nu_{1 \mathrm{x}}}{\nu_{12}}+\frac{\nu_{2 \mathrm{x}}}{\nu_{21}}+\frac{\nu_{1 \mathrm{x}}}{\nu_{12}} \cdot \frac{\nu_{2 \mathrm{x}}}{\nu_{21}}\right] \text {. }
\end{aligned}
$$

Ion continuity

$$
\frac{\mathrm{d}}{\mathrm{dt}}\left[\begin{array}{l}
N_{1} \\
N_{2}
\end{array}\right]=\left[\begin{array}{c}
P_{1} \\
0
\end{array}\right]-\hat{L} \cdot\left[\begin{array}{l}
N_{1} \\
N_{2}
\end{array}\right]-\nabla \cdot\left[\begin{array}{l}
\phi_{1} \\
\phi_{2}
\end{array}\right]
$$


where $P_{1}=\mathrm{O}^{+}$photoionization frequency

$N_{\mathrm{e}} / N_{1} / N_{2}=$ electron $/ \mathrm{O}^{+} / \mathrm{H}^{+}$density

$T_{\mathrm{e}} / T_{1} / T_{2}=$ temperatures

$U_{\mathrm{e}} / U_{1} / U_{2}=$ average drift velocities

$q_{\mathrm{e}} / q_{\mathrm{i}}=$ electron/ion heat flux

$\Sigma Q_{\mathrm{e}} / \Sigma L_{\mathrm{e}}=$ sum of electron heating/cooling rates

and we have used the thermal conductivity coefficients $\left(\lambda_{i}, \lambda_{e}\right)$, the diffusion coefficients $\left(D_{i}\right)$ and terms in the diffusive force, which we symbolize by $Q_{i}$, from St. Maurice and Schunk (1977). The matrix $\hat{L}$ contains the chemical reaction frequencies including the $\mathrm{H}^{+}+\mathrm{O}$ charge exchange reaction, the reverse reaction and all other ion neutral reactions. The term $\xi$ in the denominator of (5) is given by St. Maurice and Schunk as

$$
\xi=\nu_{\mathrm{ij}}{ }^{\prime} \nu_{\mathrm{ji}}{ }^{\prime} /\left(\nu_{\mathrm{i}}{ }^{\prime}-\nu_{\mathrm{j}}{ }^{\prime}\right),
$$

where the $\nu_{\mathrm{ji}}{ }^{\prime}, \nu_{\mathrm{ij}}{ }^{\prime}, \nu_{\mathrm{i}}{ }^{\prime}$ and $\nu_{\mathrm{j}}{ }^{\prime}$ are their "effective collision frequencies." Also we may collect the diffusive force terms given by them in the form

$$
\begin{aligned}
Q_{\mathrm{i}}=\frac{\nabla\left(N_{\mathrm{i}} T_{\mathrm{i}}\right)}{N_{\mathrm{i}} T_{\mathrm{i}}} & -\frac{m_{\mathrm{i}} g_{\|}}{\mathrm{k} T_{\mathrm{i}}}+Z_{\mathrm{i}} \frac{T_{\mathrm{e}}}{T_{\mathrm{i}}} \frac{\nabla\left(N_{\mathrm{e}} T_{\mathrm{e}}\right)}{N_{\mathrm{e}} T_{\mathrm{e}}} \\
& \mp \gamma_{\mathrm{i}} \frac{\nabla T_{\mathrm{e}}}{T_{\mathrm{i}}} \pm \frac{N_{\mathrm{j}}}{N_{\mathrm{e}}}\left[\frac{\alpha_{\mathrm{ij}}}{T_{\mathrm{i}}} \nabla T_{\mathrm{i}}-\frac{\alpha_{\mathrm{ij}}{ }^{*}}{T_{\mathrm{i}}} \nabla T_{\mathrm{j}}\right],
\end{aligned}
$$

where the upper/lower sign applies to the $\mathrm{O}^{+} / \mathrm{H}^{+}$; $\alpha_{\mathrm{ij}}, \alpha_{\mathrm{ij}}{ }^{*}$ and $\alpha_{\mathrm{i}}$ are thermal diffusion coefficients and $D_{\mathrm{i}}$ is the ordinary ion diffusion coefficient of species $i$, as in St. Maurice and Schunk (1977).

\section{NUMERICAL SOLUTION}

\section{Outline of approach}

Equations (3-8) form a system of 8 first order differential equations. Equations (5) and (6) can be substituted into (4) and (3) respectively to form two parabolic partial differential equations. Similarly, equation (7) can be substituted into (8) to yield 2 parabolic equations. This substitution is done numerically. The two lower boundary conditions required for each parabolic equation are supplied at the ends of the field tube. The lower boundary altitude can be chosen to ensure that local equilibrium conditions prevail. This approach has worked successfully for the solution of the energy equations. In the case of the continuity equations, numerical problems are encountered above some altitude which generally lies between 1000 and $3000 \mathrm{~km}$ depending on prevalent conditions. In this region the diffusion coefficient, $D_{\mathrm{i}}$, becomes very large although the ion fluxes remain finite. Therefore the diffusive force term, $Q_{i}$, given by (10) tends to zero although the individual terms of $Q_{i}$ do not become small at high altitudes. The fractional error of $Q_{i}$ becomes large at high altitudes, and so does the fractional errors of ion flux, which is a linear combination of $Q_{i}$ terms when calculated from the mementum equations. We therefore use equation (10) to compute the $\mathrm{O}^{\prime}$ and $\mathrm{H}^{\prime}$ densities in the region where $Q_{\mathrm{i}} \approx 0$.

We divide the plasma flux tube into three regions $\mathrm{B}, \mathrm{C}$, and $\mathrm{B}^{*}$ shown in Fig. 1. In the low altitude regions $B$ and $B^{*}$ both ion-ion and ion-neutral collisions are important and the full parabolic equations are used. These equations accurately reproduce the plasma concentration and flow in this region. In region $\mathrm{C}$, the relative flow terms become insignificant for $\mathrm{H}^{+}$and $\mathrm{O}^{+}$in the plasmasphere and the diffusive equilibrium approximation accurately describes the distribution of plasma along the field tube in this region. The solutions for each region are coupled through a flux preserving approach which is described in detail in subsequent sections. The approach eliminates the low altitude problems which limit the parabolic approach.

Figure 2 schematically illustrates the model used in the computation. An effective simultaneous solution to this scheme is obtained as follows. The continuity/momentum and electron-ion energy equations are solved in a ping-pong iterative mode using density and temperature profiles taken from the previous time step to predict values at the current time step. In practice the predicted current values lie so close to the final solution values that use of the predictive technique renders the solution of the density, temperature and flow equations effectively simultaneous.

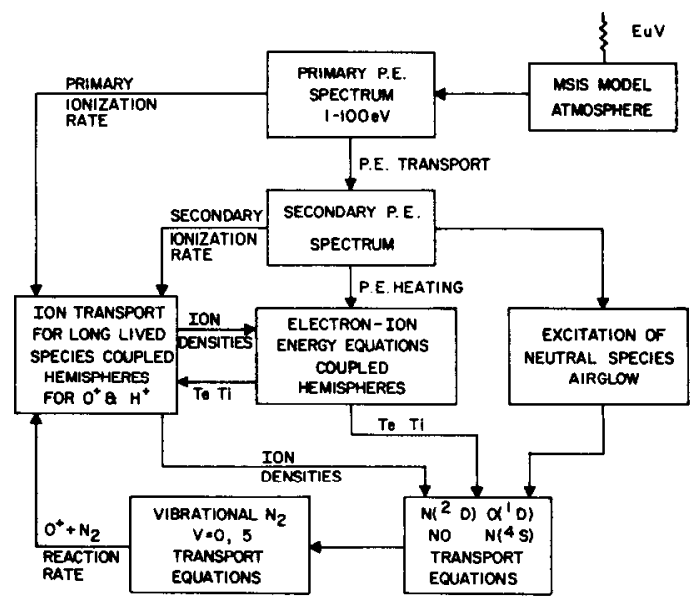

Fig. 2. Overall SChematic OF THE MODEL. 


\section{The flux preserving method}

Our system of equations comprise 4 second order partial differentials. The flux preserving method that we use to solve the equations involves a Newton iteration similar to that described by Hastings and Roble (1977) (see Appendix B). The main difference between the flux preserving approach and earlier methods lies in our choice of the minimization function $F$ given by equation B2 in Appendix B.

As will beconte evident from the discussion that follows, the most suitable choice of $F$ to use with variable spatial steps, is an integral form of the coupled parabolic density equations. The integration is carried out between limits located midway between grid points, e.g. if the function at the $k$ th point is denoted by $F_{\mathrm{k}}$, the integration is carried out between points $k-\frac{1}{2}$ and $k+\frac{1}{2}$, as illustrated in Fig. 3, i.e.

$$
\begin{aligned}
{\left[\begin{array}{l}
F_{1} \\
F_{2}
\end{array}\right]_{\mathrm{k}}=\left.\frac{1}{B(S)}\left[\begin{array}{l}
\phi_{1} \\
\phi_{2}
\end{array}\right]\right|_{S_{\mathrm{k}-1 / 2}} ^{S_{\mathrm{k}+1 / 2}}-\int_{S_{\mathrm{k}-1 / 2}}^{S_{\mathrm{k}+1 / 2}}\left\{\left[\begin{array}{c}
P_{1} \\
0
\end{array}\right]\right.} \\
\left.-\hat{L} \cdot\left[\begin{array}{l}
N_{1} \\
N_{2}
\end{array}\right]-\frac{\partial}{\partial t}\left[\frac{N_{1}}{N_{2}}\right]\right\} \frac{\mathrm{d} s}{B(S)}=0 .
\end{aligned}
$$

Equation (12) is just the matrix integral form of the coupled ion continuity equations. When the substitution for $\left[\begin{array}{l}\phi_{1} \\ \phi_{2}\end{array}\right]$ is made from equation (7) their parabolic form is clear. The integration is carried out numerically with (11) expressed in second order finite difference form as discussed in the next section. Between grid points in regions $B$ or $\mathrm{B}^{*}$ densities are interpolated linearly in evaluating (11). This approach lends itself naturally to the use of integration steps of arbitrary size. The approach may be termed a "flux preserving method." The use of an integral form of the continuity equation, while retaining the second order finite differencing, crucially simplifies the inclusion of region $\mathrm{C}$ in an iterative solution scheme. In this case we integrate from boundary 2 to boundary $2^{*}$, across the entire region $\mathrm{C}$, whereas in the region $\mathrm{B}$ we integrate between grid points $k-\frac{1}{2}$ and $k+\frac{1}{2}$ as discussed above.

$$
\begin{aligned}
& {\left[\begin{array}{l}
F_{1} \\
F_{2}
\end{array}\right]=-\left\{\frac{1}{B\left(2^{*}\right)}\left[\begin{array}{l}
\phi_{1}\left(2^{*}\right) \\
\phi_{2}\left(2^{*}\right)
\end{array}\right]-\frac{1}{B(2)}\left[\begin{array}{l}
\phi_{1}(2) \\
\phi_{2}(2)
\end{array}\right]\right\}} \\
& +\int_{2}^{2^{*}}\left\{\left[\begin{array}{c}
P_{1} \\
0
\end{array}\right]-L \cdot\left[\begin{array}{l}
N_{1}(S) \\
N_{2}(s)
\end{array}\right]\right. \\
& \left.-\frac{\partial}{\partial t}\left[\begin{array}{l}
N_{1}(S) \\
N_{2}(S)
\end{array}\right]\right\} \frac{\mathrm{d} s}{B(S)} .
\end{aligned}
$$

In region $C$ the densities at the many grid points between boundaries 2 and $2^{*}$ are no longer interpolated linearly as is done in region $\mathbf{B}$, but are calculated from the densities at point 2 via numerical integration of physically realistic equation of diffusive equilibrium. This may be expressed in functional form at some point $\mathrm{k}$ of region $\mathrm{C}$ as

$$
N_{\mathrm{i}}(\mathrm{k})=G_{\mathrm{i}}\left(N_{\mathrm{i}}(2), N_{2}(2), \mathrm{k}\right),
$$

where 2 in parenthesis refers to boundary 2 . The boundary fiuxes used in the continuity equation of region $\mathrm{C}$ are calculated via the full momentum equations which are still valid at the boundaries 2 and $2 *$. Region $\mathrm{C}$ appears as one large grid step to the code and is therefore handled in effectively the same way as any other step in region B. It is this feature which is new, and renders the solution an efficient method of solving the interhemispheric coupled equations.

\section{The finite difference scheme}

We use the following scheme to evaluate the time average of any parameter $f$, and its spatial derivative $\partial f / \partial s$ at a point midway between grid points $\mathrm{k}$ and $\mathrm{k}+1$ during the time interval $t_{1}$ to $t_{1+1}$.

$$
\begin{aligned}
f\left(S_{\mathrm{k}+1 / 2}\right)= & \frac{\theta}{2} f\left(S_{\mathrm{k}}, t_{\mathrm{l}+1}\right) \\
& +\frac{(1-\theta)}{2} f\left(S_{\mathrm{k}}, t_{\mathrm{l}}\right) \\
& +\frac{\theta}{2} f\left(S_{\mathrm{k}+1}, t_{\mathrm{l}+1}\right)+\frac{(1-\theta)}{2} f\left(S_{\mathrm{k}+1}, t_{\mathrm{l}}\right) \\
\left.\frac{\partial f}{\partial t}\right|_{\mathrm{k}+1 / 2}= & \theta \frac{\left[f\left(S_{\mathrm{k}+1}, t_{\mathrm{l}+1}\right)-f\left(S_{\mathrm{k}}, t_{\mathrm{l}+1}\right)\right]}{S_{\mathrm{k}+1}-S_{\mathrm{k}}} \\
& +(1-\theta) \frac{\left[f\left(S_{\mathrm{k}+1}, t_{\mathrm{l}}\right)-f\left(S_{\mathrm{k}}, t_{\mathrm{l}}\right)\right]}{S_{\mathrm{k}+1}-S_{\mathrm{k}}}
\end{aligned}
$$

The parameter $\theta$ provides the option to select the finite difference scheme, with $\theta=\frac{1}{2}$ for CrankNicholson and $\theta=1$ for Laasonen differencing, or any value in between.

Referring to Fig. 3, we see that the computation of equation (11), totally within one of the regions $B$ or $\mathrm{B}^{*}$ is based upon the ion densities at three adjacent grid points, each one of which receives an independent correction in the iterative solution. Linear interpolation is used to interpolate the densities between the three consecutive grid points. The computation of equation (12) on the other hand involves a number of dependent densities at intervening points as well as the densities at points 2B, 2A and $2 \mathrm{~B}^{*}$ as was discussed above. The boundary fluxes $\phi(2)$ and $\phi\left(2^{*}\right)$ are each calculated from the densities at the adjacent point pairs (2B, 


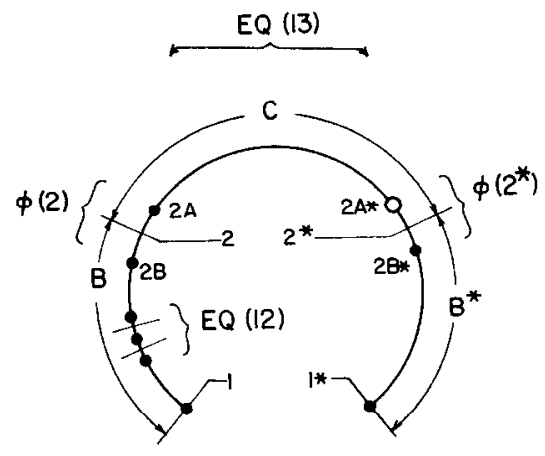

Fig. 3. Schematic of SPATIAL GRID.

Boundaries 2 and $2^{*}$ are each straddled by the pairs of grid points $(2 \mathrm{~A}, 2 \mathrm{~B})$ and $\left(2 \mathrm{~A}^{*}, 2 \mathrm{~B}^{*}\right)$ respectively, from which the boundary fluxes are calculated. The boundaries 1 and $1^{*}$ on the other hand fall exactly upon the first and last point. The densities at these points are calculated via local photochemical equilibrium. Densities are needed at 3 points to calculate each finite difference equation used in the Newton Raphson Iterative solution. The three points are adjacent for equation 12 but not for equation 13. The three points needed to calculate equation 13 are $2 \mathrm{~B}, 2 \mathrm{~A}$ and $2 \mathrm{~B}^{*}$.

$2 \mathrm{~A})$ and $\left(2 \mathrm{~A}^{*}, 2 \mathrm{~B}^{*}\right)$ respectively. It is to be noticed however that the ion densities at point $2 \mathrm{~A}^{*}$ are not independent, but computed via the equations of diffusive equilibrium from the densities at point 2A. The integral terms also involve dependent ion densities at all the grid points between $2 \mathrm{~A}$ and $2 \mathrm{~B}^{*}$.

In summary, even though equation (12) involves all the grid points of region $\mathrm{C}$, only the densities at the points $2 \mathrm{~B}, 2 \mathrm{~A}$ and $2 \mathrm{~B}^{*}$ receive independent corrections during the iterative solution procedure. In principle the entire region $\mathrm{C}$ acts as a single "heavy point," keyed to the density at $2 \Lambda$, in the Newton-Raphson solution of the density equations, as was stated earlier.

The code may be run with Crank-Nicholson, implicit or any type of intermediate finite differencing scheme under the control of the implicit fraction parameter. Thus if $\theta$ is input, $N_{\mathrm{j}}$ would be approximated as $\theta N_{\mathrm{j}}(t+\Delta t)+(1-\theta) N_{\mathrm{j}}(t)$ and so on. Either fully time dependent or steady state simulation may be selected via another parameter which artificially nulls the time derivative term in the ion continuity equation.

\section{TYPICAL RESULTS}

We first discuss the tests we performed to demonstrate that the simulation converges to a stable steady state solution. We then analyze the steady state ion fluxes, and finally we present simulations of the collapse of the sunset ionosphere. Numerical aspects of this work have been reported by Young et al. (1979a).

For the stability test, the rotation of the earth and the time evolution of all locally computed parameters were frozen at UT 17:33, 10 August, and furthermore the same parameters were set artificially to $\mathrm{N}-\mathrm{S}$ symmetry, at their $\mathrm{N}$. hemisphere values, about the magnetic equator of an $L=2$ field line at approximately $70 \mathrm{~W}$ longitude. Our temperature model for this test featured $T_{\mathrm{i}}=4500 \mathrm{~K}, T_{\mathrm{e}}=$ $5500 \mathrm{~K}$ at the magnetic equator and $T_{\mathrm{i}}=500 \mathrm{~K}$, $T_{\mathrm{e}}=550 \mathrm{~K}$ at $120 \mathrm{~km}$. Our initial temperature profiles were also artificially symmetric. To obtain a steady state solution the time derivative terms of equations (11) and (12) were set equal to zero and the simulation allowed to iterate to convergence.

Using the steady state solution as an initial condition we then allowed the simulation to run in the time dependent mode, but with all the local ambient parameters still frozen at UT 17:33. Using many $5 \mathrm{~min}$ time steps we found that the time dependent density profile simulation did not drift and displayed an oscillation of less than one part in a million about the steady state solution. The profiles also proved to be symmetric to better than one part in a million. We feel that the results of these tests constitute a rigorous test of stability and conservation of particles (Young et al., 1979a).

The steady state symmetric profiles show some interesting aspects of ion production and flow which indicate that the code is reproducing expected geophysical variations. The steady state flux profiles, denoted $0 \mathrm{~h}$ in Fig. 4, show downflow of both $\mathrm{O}^{+}$and $\mathrm{H}^{+}$below $500 \mathrm{~km}$ but counterstreaming above with $\mathrm{O}^{+}$moving upwards and $\mathrm{H}^{+}$downwards. The $\mathrm{O}^{+}$downflux reaches a maximum value near the altitude of the $\mathrm{O}^{+}$density peak at about $250 \mathrm{~km}$ as shown in Fig. 5. This can be attributed to the fact that the chemical loss rate, due to reaction of $\mathrm{O}^{+}$with $\mathrm{N}_{2}$ and $\mathrm{O}_{2}$ increases with decreasing altitude more rapidly than the photoionization production rate as one approaches the $\mathrm{O}^{+}$density peak from above.

Above about $500 \mathrm{~km}$, the calculated $\mathrm{O}^{+}$flux is upward to replace charge exchanged $\mathrm{O}^{+}$as is typically seen in daytime measurements (Evans and Holt, 1978), and the $\mathrm{H}^{+}$is counterstreaming downward. At altitudes above $550 \mathrm{~km}$, the counterstreaming $\mathrm{H}^{+}$and $\mathrm{O}^{+}$are virtually equal in magnitude except for a small residual difference caused by the small amount of photoproduction even at very high altitudes.

Photoproduction and reactions with molecular neutrals are very small above $550 \mathrm{~km}$. Under 


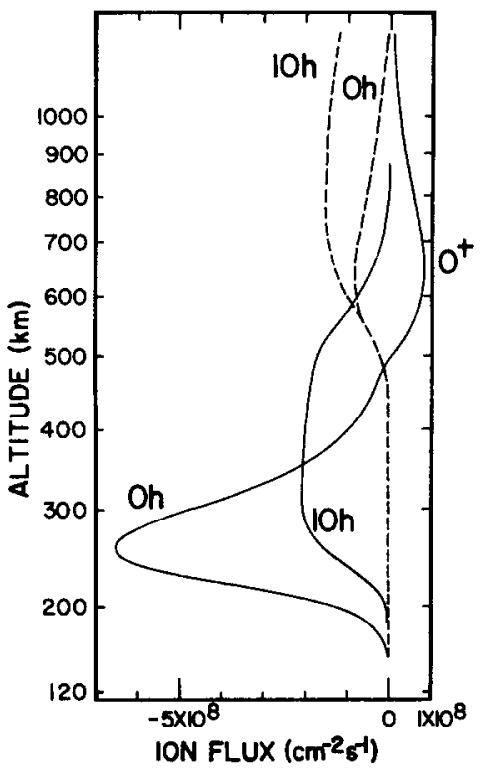

Fig. 4. Simulated ION fluXes as a FUNCTION OF ALTITUDE ALONG THE $L=2$ FIELD TUBE.

The full lines are for $\mathrm{O}^{+}$and the dashed curves, $\mathrm{H}^{+} .0 \mathrm{~h}$ denotes steady state conditions, and $10 \mathrm{~h}$ represents $10 \mathrm{~h}$ after sunset.



Fig. 5. Simulated ION DENSTTIES AS A FUNCTION OF ALTITUDE ALONG THE $L=2$ FIELD TUBE.

For $\mathrm{H}^{+}$curves for steady state conditions and ten hours after the collapse denoted $0 \mathrm{~h}$ and $10 \mathrm{~h}$ respectively are shown. For $\mathrm{H}^{+}$the curves denoted $0,1,3$ and $10 \mathrm{~h}$ denote steady state and then one, three and ten hours after the simulated ionospheric collapse. steady state conditions $\partial N_{\mathrm{i}} / \partial t=0$ as well, so with the artificially symmetric conditions, $\phi_{\mathrm{H}^{+}}(S)+$ $\phi_{\mathrm{O}^{+}}(S)=0$ by conservation of charge. In a separate work, Young et al. (1979b) have shown that quite simply, counterstreaming is required by the continuity equations. The particular direction of the counterstreaming being determined by the scale heights of the two products $[\mathrm{H}]\left[\mathrm{O}^{+}\right]$and $\left[\mathrm{H}^{+}\right][\mathrm{O}]$ (Young et al., 1979b) at higher and lower altitudes. The counterstreaming of $\mathrm{H}^{+}$and $\mathrm{O}^{+}$is a fundamental result for steady state symmetric simulations with closed magnetic flux tube models which do not allow any cross field movement of ions.

Under time dependent conditions $\partial N_{\mathrm{i}} / \partial t \neq 0$. Photoproduction and reactions with molecular neutrals remain small at high altitudes. If we average over a time interval we find:

$$
\left\langle\nabla \cdot \phi_{\mathrm{H}^{+}}\right\rangle+\left\langle\nabla \cdot \phi_{\mathrm{O}^{+}}\right\rangle=-\frac{\int_{0}^{\tau}\left(\frac{\partial \mathrm{N}_{1}}{\partial \mathrm{t}}+\frac{\partial N_{2}}{\partial t}\right) \mathrm{d} t}{\tau} .
$$

The right hand side of equation (17) approaches zero as $\tau$ is increased. If artificial N-S symmetry is still maintained we retain the following weaker statement about average fluxes (Young et al., 1979b).

$$
\left\langle\phi_{\mathbf{H}^{+}}(S)\right\rangle=-\left\langle\phi_{\mathbf{O}^{+}}(S)\right\rangle .
$$

And in non-symmetric time dependent cases the locally valid equation is further weakened to a statement conserving flux at both ends of any bounded portion of a field line (Young et al., 1979b).

$$
\begin{aligned}
\left(\left\langle\phi_{\mathbf{H}^{+}}(\mathrm{b})\right\rangle\right. & \left.-\left\langle\phi_{\mathbf{H}^{+}}(\mathbf{a})\right\rangle\right) \\
& =-\left(\left\langle\phi_{\mathbf{O}^{+}}(\mathbf{b})\right\rangle-\left\langle\phi_{\mathrm{O}^{+}}(\mathrm{b})\right\rangle-\left\langle\phi_{\mathrm{O}^{+}}(\mathbf{a})\right\rangle\right),
\end{aligned}
$$

where $a$ and $b$ are two separate points on the field line.

Although the counterstreaming of $\mathrm{H}^{+}$and $\mathrm{O}^{+}$is a fundamental result applying to any model of a closed plasmaspheric flux tube allowing field aligned transport only, the effect has been widely overlooked in the literature because it is masked for the most part by diurnal variations. Time averaging would be needed to deconvolve the counterstreaming under realistic conditions. Vickrey et al. (1979) observed counterstreaming during twilight conditions at Arecibo when the common diurnal component of ion flow was on the point of reversing itself. Bailey et al. (1977) confirmed that counterstreaming is also predicted theoretically at twilight. Careful measurement of the amount by which (17) and (18) are not satisfied experimentally 
would be a measure of the total amount of cross field ion diffusion which will eventually be needed in a truly accurate model of the plasmasphere (Young et al., 1979b).

We have also simulated an ionospheric collapse, as might occur at sunset. For this purpose we allowed the electron heating rate and the photoproduction rate to decay exponentially at each point with a time constant of $20 \mathrm{~min}$. The results are shown in Fig. 5 as curves annotated with the time after the initiation of collapse. Times of $0,1,3$ and $10 \mathrm{~h}$ are shown for $\mathrm{O}^{+}$, while only 0 and $10 \mathrm{~h}$ are shown, for clarity of display, in the case of $\mathrm{H}^{+}$.

The collapse of the topside $\mathrm{O}^{+}$density profile is the most profound sunset effect, while the topside $\mathrm{H}^{+}$density profile only appears to settle slightly. Both effects however are due to the same cause: the reduction of the topside ion and electron temperatures. In our temperature model, the equatorial temperatures are both reduced to about $1100 \mathrm{~K}$ after $10 \mathrm{~h}$ from initial values of $T_{\mathrm{i}}=4500 \mathrm{~K}$ and $T_{e}=5500 \mathrm{~K}$ used for the steady state. The concomitant reduction in topside scale heights causes the $\mathrm{O}^{+}$density, which had a shorter scale height to begin with, to decrease more rapidly.

It will be noticed that the reduction of densities is moderate at middle altitudes and then again very pronounced near the lower boundary of our simulation. Temperature effects have less influence at low altitudes. The more dramatic decrease there is to be expected however, because the chemical destruction rate is much greater than at the middle altitudes.

The counterstreaming reported for steady state conditions disappears, to be replaced within about one hour by downstreaming of both ions everywhere. Even after $10 \mathrm{~h}$ however. there remains a residual pattern that looks as if the steady state counterstreaming were superimposed upon a new downflux. It would appear that the profiles are still in the process of adjusting to the reduced temperatures. The reduced photoproduction can no longer support an upflux of $\mathrm{O}^{+}$at high altitudes in any case.

The $\mathrm{H}^{+}-\mathrm{O}^{+}$transition height is also lowered at night. Where it was $1150 \mathrm{~km}$ under daytime steady state conditions it drops to only $650 \mathrm{~km}$ after $10 \mathrm{~h}$. The lower transition height is a combined effect resulting from both the reduction of the neutral and ion scale heights under the lower temperature post collapse conditions and the new downflow of ions at that time. Note that the $\mathbf{H}^{+}$density actually increases at around $800 \mathrm{~km}$. The basic features agree with the geophysical observations of Evans and Holt (1978) and demonstrate clearly that the code is working properly.

\section{TEMPERATURE RESULTS}

The model has also been used to simulate experimental data from the S3-3 satellite, for orbit number 1035 (Rich et al., 1979). A comparison between the theoretical electron temperature and measurements is shown in Fig. 6.

The most significant feature of the comparison is the good agreement between the theoretical and experimental electron temperature gradients. It should be noted that there is considerably more scatter in the temperature measurements than in the gradient derived from them. Previous measurements have indicated the existence of large temperature gradients in the magnetosphere (Brace, 1970; Serbu and Maier, 1967). Such large gradients are inconsistent with the classical thermal conductivity of Spitzer, which has been used in our model, and which produces small gradients at high altitudes. Mayr and Volland (1968) have shown that outside the plasmapause where densities are very small, the thermal heat flow is greatly reduced. On the other hand, Mayr et al. (1973) showed that within the plasmasphere the thermal conductivity is only slightly changed from the classical theory. We have chosen to use the classical form of the thermal conductivity until further theoretical work produces a different result.

Although the theoretical temperatures in Fig. 6 are within the error bounds of the S3-3 results, such high temperatures were only obtained by including an extra heat source in the protonosphere.

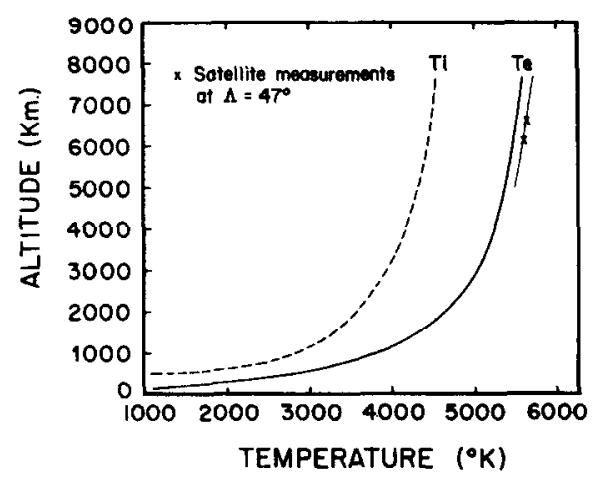

Fig. 6. THE RESULTS OF A THEORETTCAL SIMULATION OF THE S3-3 SATELLTE $T_{e}$ MEASUREMENTS FOR ORBIT NUMBER 1035.

The theoretical results have been obtained through a steady state solution of the energy equations. 


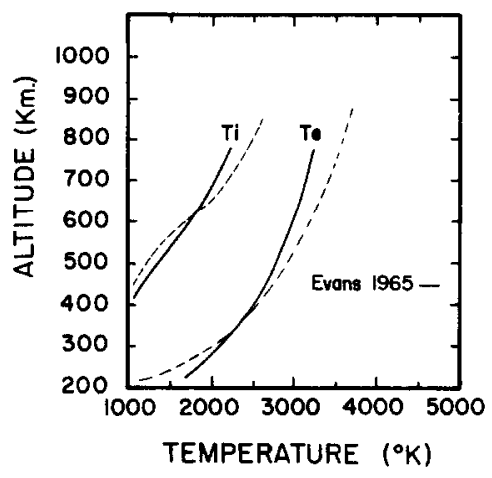

Fig. 7. COMparison of THEORETICAL $T_{i}$ AND $T_{\text {e }}$ RESUlts WITH THE DATA OF EVANS (1967) AT LOW AlTITUDES.

For even under the assumption that all photoelectrons leaving the ionosphere lose their heat in the protonosphere, the simulated electron temperature was $400 \mathrm{~K}$ lower than the data. The need to include an extra heat source may be due to the calculated photoelectron fluxes being too small. However, the question is not yet resolved, and there is still a possibility that an extra heat source, such as waveparticle interactions may need to be included.

A further indication that the classical theory is possibly adequate, is that the model not only reproduces high altitude temperatures, but low altitude temperatures compare favorably with typical measurements. This can be seen in Fig. 7 where the low altitude theoretical temperatures are plotted alongside 1964 Millstone Hill data (Evans, 1967). The theoretical temperatures lie for the most part within $10 \%$ of the measurements i.e. they fall well within the day-to-day range of variability indicated by the data.

The time-dependent capabilities of the program are illustrated in Fig. 8. Starting with the conditions



Fig. 8. ION AND ELECTRON TEMPERATURE DECAY, STARTING WITH THE CONDITIONS OF FIG. 1 ANU SWTTCHING OFF THE HEATING SOURCE.

The curves are given at hourly intervals. indicated by Fig. 6 , the heating rate was reduced to simulate the decay of electron temperature at night. During the day, temperature changes are small, but during the night the temperature decays very rapidly at low altitudes and less rapidly at high altitudes where heat conduction is important and local heat loss is small.

It can be seen from Fig. 8, that although $T_{\mathrm{e}}>T_{\mathrm{i}}$ during the day, at night $T_{\mathrm{i}}>T_{\mathrm{e}}$ at high altitudes after less than an hour, because electrons lose heat by conduction faster than ions. (The curves in Fig. 8 represent hourly intervals). Figure 8 also shows that the rate of decay of electron temperature decreases with time as would be expected because of the high temperature dependence of the thermal conductivity (Nagy et al., 1968). The temperature results shown here are similar to those obtained by Richards and Cole (1979) using the shooting method. However, the above results are more accurate because the solution of the equations is simultaneous, and because the whole field line has been simulated rather than assuming zero heat flux at the equatorial plane.

\section{COMPARISON OF MODEL WITH AE DATA}

Figure 9 gives a comparison between $\mathrm{AE} \mathrm{O}^{+}$ density data and that calculated from the model. The data were taken from orbit 2758 of the AE-C satellite, near 12.77 hours UT.

Agreement in the altitude range $200-400 \mathrm{~km}$ is good but above $400 \mathrm{~km}$ agreement is very poor. Comparison between satellite data and the model is complicated by the latitudinal variation of the data. During this pass the high altitude was taken near

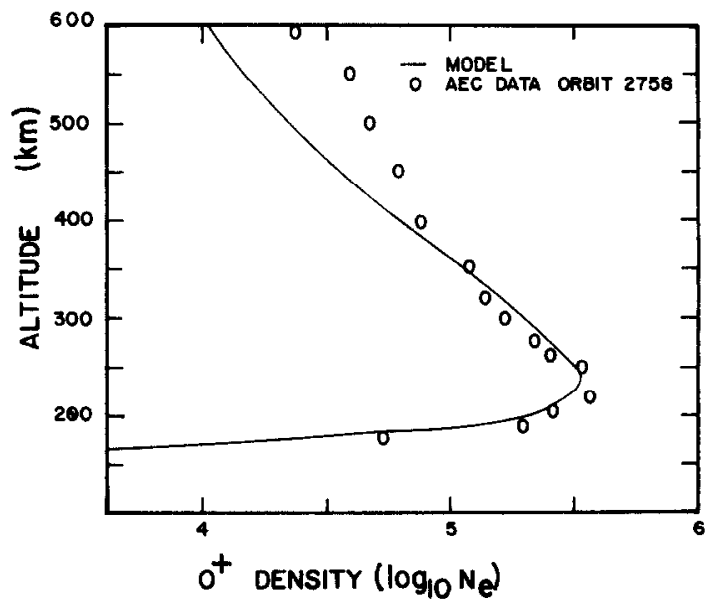

FIG. 9. COMPARISON BETWEEN MODEL AND $\mathrm{O}^{+}$DENSITIES FOR AE-C ORBIT NUMBER 2758. 


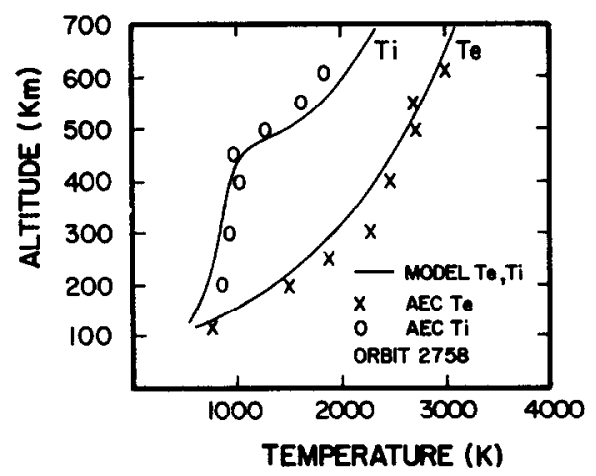

Fig. 10. COMParison BetWEen MODEI AND AE-C. ION AND ELECTRON TEMPERATURES FOR AE-C ORBTT NUMBER 2758.

$L=3.5$ while the low altitude data was taken near $L=1.5$. The differences between data and theory could be due to latitudinal gradients of density.

The electron and ion temperatures along orbit 2758 are compared in Fig. 10. The agreement in this case is much better than for the density.

\section{FUTURE IMPROVEMENIS TO THE SIMULATION}

Our simulated density profiles show that our approximation of diffusive cquilibrium is satisfactory for $\mathrm{H}^{+}$and $\mathrm{O}^{+}$above the altitude where $\mathrm{H}^{+}$ becomes the only major ion. Nevertheless there is an almost imperceptible discontinuity in the density gradients at boundaries 2 and $2^{*}$, so we intend to include ion-ion collisions at the very high altitudes at a later date. Two possible ways to do this present themselves. One is to formulate $\mathrm{O}^{+}$density as a parabolic equation at all altitudes (regions $\mathrm{B}, \mathrm{C}$ and $\mathrm{B}^{*}$ ), but retain first order momentum and continuity equations for $\mathrm{H}^{+}$at high altitudes (region C). Another way is to upgrade the diffusive equilibrium solutions iteratively by calculating each $V_{i}$ from the continuity equation integrated between boundary 2 and $2^{*}$, and then substituting it into the relative flow terms of the full momentum equation

$$
\begin{aligned}
V_{\mathrm{i}}(S)=\frac{B(S)}{N_{\mathrm{i}}(S)}\{ & \frac{\phi_{\mathrm{i}}(2)}{B(2)} \\
& \left.+\int_{2}^{\mathrm{S}}\left[P_{\mathrm{i}}-\hat{L}_{\mathrm{ij}} N_{\mathrm{j}}-\frac{\partial N_{\mathrm{i}}}{\partial t}\right] \frac{\mathrm{d} s}{B(S)}\right\}, \\
Q_{\mathrm{i}}= & -\frac{1}{D_{\mathrm{i}}}\left[\left(V_{\mathrm{i}}-V_{\mathrm{j}}\right)+\frac{\nu_{\mathrm{ix}}}{\nu_{\mathrm{ij}}} V_{\mathrm{i}}\right] .
\end{aligned}
$$

The velocity $V_{\mathrm{i}}$ in equation (20) would be calculated from the previous iteration. Note that $Q_{i}$ will now be equal to some small correction number, rather than identically equal to zero, as used in the diffusive equilibrium approximation.

A number of questions concerning tube filling, formation of the plasmasphere, plasmaspheric heat sources, ion fluxes and the maintenance of the night-time ionosphere, will form the basis for our continuing investigations.

\section{CONCLUSIONS}

We have achieved a comprehensive simulation of field aligned plasma transport in the plasmasphere. The plasma simulation itself incorporates the best aspects of two older methods in a unified mathematical model and uses accurate geophysical parameters to produce an accurate and meaningful solution to the geophysical plasma problem.

We have shown that the solutions obtained by our code are stable and display a reasonable time development even under rapidly varying conditions such as sunset. Furthermore, comparisons with satellite and radar data have shown good agreement in a number of cases. We believe that the necessity to add ad hoc heat sources to the plasmasphere on the other hand points our the necessity to employ a more comprehensive model including wave particle interactions. We have simulated the counterstreaming of $\mathrm{H}^{+}$and $\mathrm{O}^{+}$ions at high altitudes and have shown elsewhere (Young et al., $1979 \mathrm{~b}$ ) that counterstreaming is a necessary result of any steady state symmetric model of closed field tube ion transport. The preliminary results of this model have suggested a number of interesting future investigations of ion streaming, net loss from the plasmasphere, photoelectron transport, heat conduction, and the maintenance of the night-time ionosphere.

Acknowledgements - This work was supported at the University of Michigan by Air Force Contract F19628-77-C0007, NSF Grant ATM75-03986 A01, and NASA Grant NAS5-24331, and by a grant of computer resources from the National Center for Atmospheric Research which is sponsored by the National Science Foundation. We thank Drs. J. C. G. Walker, R. W. Schunk, and J.-P. St. Maurice for helpful discussions.

\section{REFERENCES}

Bailey, G. J., Moffett, R. J. and Murphy, J. A. (1977). Planet. Space Sci. 25, 967.

Bailey, G. J., Moffett, R. J. and Murphy, J. A. (1978). Planet. Space Sci. 26, 753.

Banks, P. M. (1972). Proc. of the Joint COSPAR/ IAGA/URSI Symposium.

Banks, P. M. and Nagy, A. F. (1970). J. geophys. Res. 75, 1902.

Banks, P. M., Nagy, A. F. and Axford, W. I. (1971). Planet. Space Sci. 19, 1053. 
Banks, P. M., Schunk, R. W. and Raitt, W. J. (1976). Annual Review of Earth and Planetary Sciences, 4.

Bauer, P. (1968). Ph.d. Thesis, University of Michigan.

Baxter, R. G. (1967). Planet. Space Sci. 15, 701.

Behnke, R. A. (1970). PhD. Thesis, Rice University.

Brace, L. H. (1970). Space Res. $X$, North-Holland, Amsterdam, 633.

Burgers, J. M. (1969). Flow Equations for Composite Gases, Academic Press, New York.

Carpenter, D. I. and Park, C. G. (1973). Rev. geophys. Space Phys. 11, 133.

Carpenter, D. L. and Smith, R. L. (1964). Rev. geophys. Space Phys. 2, 415.

Chappell, C. R. (1972). Rev. geophys. Space Phys. 10, 951.

Chappell, C. R. (1975). Rev. geophys. Space Phys. 13, 872.

Crank, J., and Nicholson, P. (1947). Proc. Camb. phil. Soc. Math. phys. 43, 50.

Fvans, I. V. (1967). Planet. Space Sci. 13, 1387.

Evans, J. V. (1969). Proc. IEEE 57, 496.

Evans, J. V. (1971a). Radio Sci. 6, 609.

Evans, J. V. (1971b). Radio Sci. 6, 843.

Evans, J. V. and Holt, J. M. (1978). Planet. Space Sci. 26, 727.

Evans, J. V. Julian, R. F. and Reid, W. A. (1970). M.I.T. Linculn Lab. Rept., 477.

Geisler, J. E. (1967). J. geophys. Res. 72, 81.

Geisler, J. E. and Bowhill, S. A. (1965). Aeronomy Rept. 5., University of lllinois, Urbana.

Hagen, J. B. (1972). Nat. Astr. Ionosphere Centre, Arecibo, Puerto Rico Rept. 17.

Hanson, W. B. (1964). Electron Density Profiles in the Ionosphere and Exosphere, p. 361. Pergamon Press, New York.

Hanson, W. B. and Patterson, T. N. L. (1964). Planet. Space Sci. 12, 979.

Harvey, P. J., Torr, D. G. and Barlow, R. (1972). Internal Rept. of the S.A. Nat'l. Inst. for Telecomm. Research, Johannesburg, South Africa, TEL 107.

Hastings, J. T. and Roble, R. G. (1977). Planet. Space Sci. 25, 209.

Hedin, A. E., Salah, M. E., Evans, J. V., Reber, C. A., Newton, G. P., Spencer, N. W., Kayser, D. C., Alcayde, D., Bauer, P., Cogger, L. and McClure, P. (1977a). I. geophys. Res. 82, 2139.

Hedin, A. E., Reber, C. A., Newton, G. P., Spencer, N. W., Brinton, H. C., Mayr, H. G. and Potter, W. E. (1977b). J. geophys. Res. 82, 2148.

Hinteregger, H. E. (1978). Private Communication.

Kirby-Docken, K., Constantinedes, R., Babeu, S., Oppenheimer, M. and Victor, G. A. (1979). Atomic Data and Nuclear Data Tables, 23, 63.

Kohl, H. (1966). Electron Density Profiles in the Ionosphere and Exosphere, North-Holland, Amsterdam, 231.

Kutimskaya, N. A., Polyakov, V. M., Klimov, N. N., Kuznetosova, C. M. and Gerschengorn, G. I. (1973). Geomag. and Aeron. 13, 33.

Marubashi, K., and Grebowsky, J. M. (1976). J. geophys. Res. 81, 1700.

Massa, J. L. (1974). Ph.D. Thesis, University of Michigan.

Massa, J. L., Cicerone, R. J. and Nagy, A. F. (1974). Paper presented at the Int. Symp. Solar Terr. Phys., Sao Paulo, Brazil, Summary in Atmosphere 3, 338.

Mayr, H. G., Brace, L. H. and Dunham, G. S. (1967). $J$. geophys. Res. 72, 4391.

Mayr, H. G., Fontheim, E. G. Brace, L. H., Brinton, H.
C. and Taylor, H. A. (1972). J. atmos. terr. Phys. 34, 1659.

Mayr, H. G., Fontheim, E. G. and Mahajan, K. K. (1973). Ann. geophys. 29, 21.

Mayr, H. G. and Volland, H. (1968). J. geophys. Res, 73, 4851.

Moffett, R. J. and Murphy, J. A. (1973). Planet. Space Sci. 21, 43.

Murphy, J. A. and Moffett, R. J. (1978). Planet. Space Sci. 26, 281.

Murphy, J. A., Bailey, G. J. and Moffett, R. J. (1976). J. atmos. terr. Phys. 38, 351.

Nagy, A. F. and Banks, P. M. (1972). J. geophys. Res. 77, 4277.

Nagy, A. F., Bauer, P. and Fontheim, E. G. (1968). J. geophys. Res. 73, 6259.

Park, C. G. and Banks, P. M. (1974). J. geophys. Res. 79, 4661.

Rich, F. J., Sagalyn, R. C. and Wildman, P. J. L. (1979). J. geophys. Res. 84, 1328.

Richards, P. G. (1978). Ph.D. Thesis, La Trobe University, Australia.

Richards, P. G. and Cole, K. D. (1979). Planet. Space Sci. 27, 1343.

Roble, R. G. (1975). Planet Space Sci. 23, 1017.

Rothwell, P. (1963). Proc. Inst. Conf. Ionosphere, Inst. Phys. and Phys. Soc. London, 217.

Schunk, R. W. (1975). Planet. Space Sci. 23, 437.

Schunk, R. W. (1977). Rev. geophys. Space Phys. 15, 429.

Schunk, R. W. and Nagy, A. F. (1978). Rev. geophys. Space Phys. 16, 355.

Schunk, R. W. and Walker, J. C. G. (1972). Planet. Space Sci. 20, 581.

Schunk, R. W. and Walker, J. C. G. (1973). Planet. Space Sci. 21, 1875.

Schunk, R. W. and Watkins, D. S. (1979). Planet. Space Sci. 27, 433.

Serbu, G. P. and Maier, E. J. (1967). Space Res. VII, 527.

St. Maurice, J.-P., and Schunk, R. W. (1977). Planet. Space Sci. 25, 907.

St. Maurice, J.-P., and Torr, D. G. (1978). J. geophys. Res. 83, 969.

Stubbe, P. (1970). J. atmos. terr. Phys. 32, 865.

Torr, D. G. and Torr, M. R. (1979). J. atmos. terr. Phys. 41, 797.

Torr, M. R., Torr, D. G., Ong, R. A. and Hinteregger, H. E. (1979). Geophys. Res. Ltrs. 6, No. 10, 771.

Vasseur, G. and Waldtenfel, P. (1968). J. atmos. terr. Phys. 30, 779.

Vickrey, J. F., Swartz, W. E. and Farley, D. T. (1976). Geophys. Res. Ltrs. 3, 217.

Vickrey, J. F., Swartz, W. E. and Farley, D. T. (1979). J. geophys. Res. 84, 1310.

Young, E. R., Richards, P. G. and Torr, D. G. (1980a). J. Comp. Phys. (in press).

Young, E. R., Richards P. G., and Torr, D. G. (1979b). Geophys. Res. Lett. 6, 925.

\section{APPENDIX A}

Modified dipole coordinates

The dipole coordinates $L$ and $Y$ form a set of perpendicular coordinates defined as follows along any given magnetic meridian:

$$
\begin{aligned}
& L=R /\left(R_{\mathrm{e}} \cos ^{2} \theta\right), \\
& Y=R_{\mathrm{e}}^{2} \sin \theta / R^{2},
\end{aligned}
$$




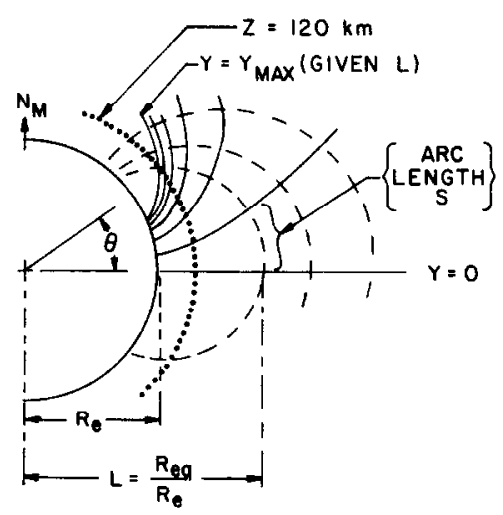

Fig. 11. Dipole and Modified Dipole CoORdinates. $L$ SHELLS: DASHED; $Y$ SHELLS: SOLID; $Z_{\text {min }}$ : DOTTED CIRCLE. Profiles of constant $Q$ or $X$ lie along the same spatial surface, but $X$ and $Q$ have different values. $S$ : arc length along the field line. $\theta$ : magnetic latitude.

where $R$ = radius from the center of the earth, $R_{\mathrm{e}}=R$ at the earth's surface, and $\theta=$ magnetic latitude, as shown in Fig. 11. $Y$ is zero on the equator, and attains its maximum, $Y_{\max }$, at the low altitude limit $Z_{\min }$ of our model. In order to allow more flexibility in point spacing we have used a modified dipole coordinate, $X$ (see Baxter, 1967) which depends upon $Y$, and a point distribution parameter $\gamma$. Thus,

$$
X=\frac{\sinh (\gamma Y)}{\sinh \left(\gamma Y_{\max }\right)}
$$

surfaces of constant $Y$ are also surfaces of even $X$, although the $Y$ and $X$ values differ. The coordinate $X$ ranges from $X=0$ at the equator to $X=1$ at the low altitude limit $Z_{\min }$. With the specification of $N-1$ equal intervals in the $X$ coordinate, we have a smoothly varying spatial step, under the control of just 2 parameters $\gamma$ and $N$.

$$
\Delta S=\Delta X\left[\frac{\sinh \left(\gamma Y_{\text {max }}\right)}{\cosh (\gamma Y)} \frac{R^{3}}{R_{\mathrm{e}}^{2}\left(1+\cos ^{2} \theta\right)^{1 / 2}}\right] .
$$

Larger values of $\gamma$ will concentrate more steps near the low altitude end of the field line while small values of $\gamma$ result in a more even spacing. $N$ specifies the total number of spatial steps. The $L, X$ coordinate system has proved sufficiently flexible to treat field lines from $L=1.5$ to $L=4$ or so with about 250 points or less.

\section{APPENDIX B}

\section{Iterative solution of differential equations}

The first step in our solution procedure is the replacement of the differential operators in each equation to be solved by the appropriate finite difference approximation (Crank and Nicholson, 1947). These methods were first introduced into the solution of aeronomical transport problems by Harvey et al. (1972) and Hastings and Roble (1977). Let a representative differential equation which is to be solved over interval $0-t$ be given by

$$
F\left[\frac{\partial n}{\partial t}, \frac{\partial^{2} n}{\partial s^{2}}, \frac{\partial h}{\partial s}, n\right]=0 .
$$

Then if $s \leq s^{k} \leq s^{L}$ represents the finite grid of $L$ points in the independent variable $s$, and an initial condition on $n$ is assumed, the differential equation becomes in principle a system of $L$ nonlinear algebraic equations in $L$ unknowns:

$$
F^{i}\left(n^{k}\right)=0,
$$

where the variable $n^{k}$ is the value of $n$ at time $t$ at point $s^{k}$, and $F^{i}$ is formed by substituting the finite difference approximation for $n$ and its derivatives at point $j$, into the formulation for $F$.

In the iterative solution one starts with some initial estimate to $n^{k}$ which in general will not be a solution of B2. However each of the dependent variables can be corrected by an amount $\delta n^{k}$ to provide a solution to B2. In order to calculate the desired vector $\delta n^{k}$ we may expand $F^{i}$ in a taylor series about the initial estimate

$$
F^{\mathrm{j}}\left(n^{\mathrm{k}}+\delta n^{\mathrm{k}}\right)=F^{\mathrm{j}}\left(n^{\mathrm{k}}\right)+J_{\mathrm{k}}^{\mathrm{j}} \delta n^{\mathrm{k}}+\frac{1}{2} K_{\mathrm{km}}{ }^{\mathrm{j}} \delta n^{\mathrm{k}} \delta n^{\mathrm{m}}
$$

where the Jacobian matrix and the second derivative matrix are:

$$
\begin{gathered}
J_{k}{ }^{j}=\frac{\partial F^{j}}{\partial n^{k}}, \\
K_{k m}{ }^{j}=\frac{\partial^{2} F^{i}}{\partial n^{k} \partial n^{m}} .
\end{gathered}
$$

In the Newton-Raphson method $K_{\mathrm{km}}{ }^{j}$ and higher terms are assumed to be insignificant. Therefore when (B3) is substituted into (B2) we have a simple linear equation in the correction vector $\delta n^{k}$

$$
F^{\mathrm{j}}\left(n^{\mathrm{k}}\right)+J_{\mathrm{k}}^{\mathrm{j}} \delta n^{\mathrm{k}}=0
$$

which is solved by multiplication by the inverse of the Jacobian matrix.

We have also evaluated the Jacobian matrix itself by a finite difference technique, rather than deriving analytic expressions (Harvey et al., 1972), wherein a small fraction $\Delta n^{k}$ of each $n^{k}$ is used to vary $F^{j}$ :

$$
J_{k}^{j}=\left[F^{j}\left(n^{k}+\Delta n^{k}\right)-F^{j}\left(n^{k}\right)\right] / \Delta n^{k}
$$

As $F^{i}$ is a very complicated function of the $n^{k}$ in this problem, the finite difference approximation to $J_{k}{ }^{j}$ has resulted in a great deal of simplification. 